\title{
Robust self-tuning regulator of time-varying linear systems with bounded external disturbances
}

\author{
Nabiha Touijer \\ Laboratory of Sciences and Techniques of \\ Automatic control and computer engineering \\ (Lab-STA), \\ National School of Engineering of Sfax, University \\ of Sfax, BP 1173, 3038 Sfax, Tunisia.
}

\author{
Samira Kamoun \\ Laboratory of Sciences and Techniques of \\ Automatic control and computer engineering \\ (Lab-STA), \\ National School of Engineering of Sfax, University \\ of Sfax, BP 1173, 3038 Sfax, Tunisia.
}

\begin{abstract}
The robust self-tuning regulator of a class of linear systems, which can be described by the input-output Auto-Regressive Moving Average with exogenous (ARMAX) mathematical model with unknown and time-varying parameters, at bounded external disturbances is developed. A scheme of polynomial approximation has been applied to approximate the unknown and time-varying parameters of systems. The modified recursive extended least squares RELS estimation algorithm with a relative dead zone is proposed and applied to estimate the unknown and time-varying parameters intervening in the ARMAX mathematical model. The formulation of the explicit schemes of self-tuning regulation problem is resolved by using the minimum variance output or the generalized minimum variance output. The obtained control law, which is an optimal solution of minimizing a correspondent criterion, permit to reduce the effect of noise upon the output of system. An example of numerical simulation illustrates the effectiveness of the explicit schemes of self-tuning regulator and presents the performances by using the modified recursive extended least squares estimation algorithm with a relative dead zone in a step of the parametric estimation of a linear time-varying systems.
\end{abstract}

\section{General terms}

Modified recursive extended least squares estimation algorithm RELS with a relative dead zone, explicit schemes of minimum variance self-tuning regulator, explicit schemes of generalized minimum variance self-tuning regulator.

\section{Key words}

Polynomial approximation; ARMAX mathematical model; time-varying parameters; modified recursive extended least squares estimation algorithm RELS with a relative dead zone; self-tuning regulator.

\section{INTRODUCTION}

A necessary stage of technologic and scientific domains (physical, chemistry, biology, economy, etc.) is the formulation of mathematical models wchich can be described the dynamic systems. The modeling permits to formalize the behavior of the dynamic systems, where we can understand, control or improve the functioning of the analyzed systems. Besides, the key in automatic control is the elaboration of dynamic system model. A procedure of estimation, which has been treated and detailed, consists to research an adequate mathematical model for any system by experimental data and available knowledge (see [9], [17] and [20]). The gotten mathematical model can be used, in the aim, of surveillance and diagnosis or for the control of the dynamic systems.

The parametric estimation of the linear dynamic systems, which can be described by input-output mathematical models with unknown and time-varying parameters, has been developed. Different types of recursive estimation algorithms, basing upon techniques of least squares, are treated and developed for resolving this problem (see [3], [8], [10], [11], [14], [15], [18], [23]).

In the literature, a schema of polynomial approximation has been proposed to track the time-varying parameters of systems ([4], [5], [12], [16], [19], [21], [22]). Applying this polynomial approximation to approximate time-varying parameters of system, a relative dead zone has been determined. In this way, a recursive estimation algorithm is used to estimate the parameters of linear dynamic system, which can be described by an input-output ARX mathematical model with unknown and time-varying parameters. This corresponds to a modified recursive least squares estimation algorithm RLS with a relative dead zone

A modified estimation algorithm RLS with dead zone, which is developed in the literature [5], doesn't permit to estimate time-varying parameters intervening in an ARMAX mathematical model, which is due to presence of immeasurable values. For this, we propose to develop a modified recursive extended lest squares estimation algorithm RELS with a relative dead zone.

The self-tuning control approach has been developed and applied to several systems [1]. Three problems of control was been an object of much research, using different schemes of self-tuning control (tracking, regulation, tracking and regulation conjointly). In the literature the algorithms developed of self-tuning regulator have been based on the recursive extended least squares method to estimate the parameters of systems. For more details on the analysis and the development of the different problems of self-tuning control, see [2], [12] and [13]. In this paper, we interest by solving the problem of regulation of linear systems, which can be described by the input-output ARMAX mathematical 
models with unknown and time-varying parameters, and with bounded external disturbances. Then, basing on the modified recursive extended least squares with a relative dead zone method to develop the algorithm of self-tuning regulator. An explicit scheme of minimum variance self-tuning regulator is developed.

The remainder of this paper is organized as follows: Section 2 treats the systems which can be described by the input-output ARMAX mathematical models with unknown and timevarying parameters with bounded external disturbances basing on a scheme of polynomial approximation to approximate the parameters of system. Then, a relative dead zone is obtained. In section 3, the problem of parametric estimation of the considered systems is solved using a proposed modified recursive extended least squares estimation algorithm with a relative dead zone. In section 4 , the problem of regulation is studied by using an explicit scheme of minimum variance self-tuning regulator. An explicit scheme of generalized minimum variance self-tuning is developed. In section 5 , a numerical simulation example illustrates the effectiveness of the control law and shown the performances of the modified recursive extended least squares estimation algorithm with a relative dead zone in a step of system parameter estimation. The last section 6 , conclusions are formulated.

\section{MATHEMATICAL MODELS}

In this paper, we interest by a parametric estimation of dynamic systems, which can be described by input-output, linear, monovariable stochastic mathematical models with unknown time-varying parameters. As the noise acting on the dynamic system is an independent random variable with zero means and constant variance.

Considering the class of linear dynamic systems with timevarying parameters, which can be described the following input-output ARMAX mathematical model:

$A\left(q^{-1}, k\right) y(k)=q^{-d} B\left(q^{-1}, k\right) u(k)+C\left(q^{-1}\right) e(k)$

where $u(k), y(k)$ and $e(k)$ represent, respectively, the input, the output and the noise of the system at the discretetime, $d$ represents the time-delay of the system, and $A\left(q^{-1}, k\right), B\left(q^{-1}, k\right)$ and $C\left(q^{-1}\right)$ are, which are defined by:

$$
\begin{aligned}
& A\left(q^{-1}, k\right)=1+a_{1}(k) q^{-1}+\ldots+a_{n_{a}}(k) q^{-n_{a}} \\
& B\left(q^{-1}, k\right)=b_{1}(k) q^{-1}+\ldots+b_{n_{b}}(k) q^{-n_{b}}
\end{aligned}
$$

and

$$
C\left(q^{-1}\right)=1+c_{1} q^{-1}+\ldots+c_{n c} q^{-n_{c}}
$$

where $n_{a}, n_{b}$ and $n_{c}$ are the orders of the polynomials $A\left(q^{-1}, k\right), B\left(q^{-1}, k\right)$ and $C\left(q^{-1}\right)$.

Noting that the parameters intervening in the polynomials $A\left(q^{-1}, k\right)$ and $B\left(q^{-1}, k\right)$ are unknown time-varying and the parameters intervening in the polynomial $C\left(q^{-1}\right)$ are unknown.

The system presented by (1) can be described by:

$y(k)=\theta^{T}(k) \varphi(k)+e(k)$ where $\theta(k)$ and $\varphi(k)$ are respectively the vector of the parameters and the vector of the observations, they are defined by:

$$
\theta^{T}(k)=\left[a_{1}(k) \ldots a_{n_{a}}(k) b_{1}(k) \ldots b_{n_{b}}(k) c_{1} \ldots c_{n_{c}}\right]
$$

and

$$
\begin{gathered}
\varphi^{T}(k)=\left[-y(k-1) \ldots-y\left(k-n_{a}\right) u(k-d-1) \ldots\right. \\
\left.u\left(k-d-n_{b}\right) e(k-1) \ldots e\left(k-n_{c}\right)\right]
\end{gathered}
$$

Basing upon the procedure of polynomial approximation treated in references [5], [16] and [21], we can applied a limited development of a function around zero to define this function in this point. For this reason, we can used a variable $\alpha_{k}$; when if $k=0, \alpha_{k}=0$ and if $k \rightarrow+\infty, \alpha_{k} \rightarrow 1$.

Then, we have the following representation, respectively, of the polynomials $A\left(q^{-1}, k\right)$ and $B\left(q^{-1}, k\right)$ :

$$
A\left(q^{-1}, k\right)=A\left(q^{-1}, \alpha_{k}\right)+\zeta_{A}\left(\alpha_{k}\right)
$$

and

$$
B\left(q^{-1}, k\right)=B\left(q^{-1}, \alpha_{k}\right)+\zeta_{B}\left(\alpha_{k}\right)
$$

with

$$
\begin{aligned}
& A\left(q^{-1}, \alpha_{k}\right)=1+\sum_{i=1}^{n_{a}} a_{i}\left(\alpha_{k}\right) q^{-i} \\
& B\left(q^{-1}, \alpha_{k}\right)=\sum_{i=1}^{n_{b}} b_{i}\left(\alpha_{k}\right) q^{-i} \\
& \zeta_{A}^{T}\left(\alpha_{k}\right)=\left[\zeta_{a_{1}}\left(\alpha_{k}\right) \ldots \zeta_{a_{n a}}\left(\alpha_{k}\right)\right]
\end{aligned}
$$

and

$$
\zeta_{B}^{T}\left(\alpha_{k}\right)=\left[\zeta_{b_{1}}\left(\alpha_{k}\right) \ldots \zeta_{b_{n b}}\left(\alpha_{k}\right)\right]
$$

we define the approximate parameters vector by:

$$
\theta_{a p}^{T}\left(\alpha_{k}\right)=\left[a_{1}\left(\alpha_{k}\right) \ldots a_{n_{a}}\left(\alpha_{k}\right) b_{1}\left(\alpha_{k}\right) \ldots b_{n_{b}}\left(\alpha_{k}\right) c_{1} \ldots c_{n_{c}}\right]
$$

when

$a_{i}\left(\alpha_{k}\right)=\sum_{j=0}^{N i} a_{i j} \ell_{j}\left(\alpha_{k}\right)$

and

$$
b_{i}\left(\alpha_{k}\right)=\sum_{j=0}^{M i} b_{i j} \ell_{j}\left(\alpha_{k}\right)
$$

where the parameters $N_{i}$ and $M_{i}$ represent, respectively, the order of limited development of $a_{i}\left(\alpha_{k}\right)$ and $b_{i}\left(\alpha_{k}\right)$, and the parameter $\ell_{j}\left(\alpha_{k}\right)$ can be calculated, when $\ell_{0}\left(\alpha_{k}\right)=1$.

The parameters vector is defined as follow:

$$
\theta(k)=\theta_{a p}\left(\alpha_{k}\right)+\zeta\left(\alpha_{k}\right)
$$


when

$$
\zeta^{T}\left(\alpha_{k}\right)=\left[\zeta_{A}^{T}\left(\alpha_{k}\right) \zeta_{B}^{T}\left(\alpha_{k}\right)\right]
$$

Equations (15) and (16) can be described again as to follow:

$$
a_{i}\left(\alpha_{k}\right)=\Gamma_{N i}\left(\alpha_{k}\right) \theta_{a_{i}}
$$

and

$b_{i}\left(\alpha_{k}\right)=\Gamma_{M i}\left(\alpha_{k}\right) \theta_{b_{i}}$

with

$\theta_{a_{i}}^{T}=\left[a_{i 0}, \ldots, a_{i N_{i}}\right]$

$\theta_{b_{i}}^{T}=\left[b_{i 0}, \ldots, b_{i M_{i}}\right]$

$\Gamma_{N i}\left(\alpha_{k}\right)=\left[1 \ell_{1}\left(\alpha_{k}\right) \ldots \ell_{N_{i}}\left(\alpha_{k}\right)\right]$

and

$\Gamma_{M i}\left(\alpha_{k}\right)=\left[1 \ell_{1}\left(\alpha_{k}\right) \ldots \ell_{M_{i}}\left(\alpha_{k}\right)\right]$

Combining (19) and (20) in (14), we obtain:

$\theta_{a p}\left(\alpha_{k}\right)=\Gamma\left(\alpha_{k}\right) \theta_{e}$

when

$$
\begin{aligned}
\Gamma\left(\alpha_{k}\right)=\operatorname{diag}[ & \Gamma_{N_{1}}\left(\alpha_{k}\right), \ldots, \Gamma_{N n a}\left(\alpha_{k}\right), \\
& \left.\Gamma_{M_{1}}\left(\alpha_{k}\right), \ldots, \Gamma_{M n b}\left(\alpha_{k}\right), I_{n c \times n c}\right]
\end{aligned}
$$

and

$\theta_{e}^{T}=\left[\theta_{a_{1}}^{T}, \ldots, \theta_{a_{n a}}^{T}, \theta_{b_{1}}^{T}, \ldots, \theta_{b_{n b}}^{T}, c_{1}, \ldots, c_{n c}\right]$

Then, the parameters vector is defined by:

$\theta(k)=\Gamma\left(\alpha_{k}\right) \theta_{e}+\zeta\left(\alpha_{k}\right)$

The dynamic system given by (5) can be represented by the following expression:

$y(k)=\theta_{e}^{T} X(k)+v(k)$

where

$X(k)=\Gamma\left(\alpha_{k}\right)^{T} \varphi(k)$

and

$v(k)=\zeta^{T}\left(\alpha_{k}\right) \varphi(k)+e(k)$

Hypothesis a: an upper bound $\rho$ of $\zeta\left(\alpha_{k}\right)$ is known [5].

Hypothesis $b$ : $e(k)$ is a bounded and an upper bound $m_{0}$ of $e(k)$ is known [5].

Basing on the hypothesis $a$ and $b$, the upper bound $d(k)$ of $v(k)$ is given by:

$d(k)=\rho\|\varphi(k)\|+m_{0}$
The precedent function will be used to give a relative dead zone in the recursive adaptive algorithm.

\section{PARAMETER ESTIMATION}

The parametric estimation of the stochastic dynamic system with time-varying parameters, which is defined by (29), can be made using a modified estimation algorithm RLS with relative dead zone were [5]:

$\hat{\theta}_{e}(k)=\hat{\theta}_{e}(k-1)+\delta(k) L(k) \xi(k)$

$L(k)=\frac{P(k-1) X(k)}{\mu(k)+X^{T}(k) P(k-1) X(k)}$

$P(k)=[I-\delta(k) L(k) X(k)] P(k-1)$

$\xi(k)=y(k)-\hat{\theta}_{e}^{T}(k-1) X(k)$

$\delta(k)= \begin{cases}0 & \text { if }|\xi(k)| \leq \beta d(k) \\ \gamma & \text { otherwise } \gamma \in[\sigma, 1 / \beta-\sigma]\end{cases}$

The constants $\sigma \in(0,1), \beta>2 / 1+\sigma$ and $\mu(k) \in\left[\begin{array}{ll}0 & 1\end{array}\right]$ are chosen arbitrarily by the user, see [5].

But, the using of this algorithm (33) can be lead to a failure. The causes are the presence of non measurable variable $\left(e(k-p) ; p=1, \ldots, n_{c}\right)$ in the vector of observations $X(k)$. In this way, the using of their a priori prediction values $\left(\varepsilon(k-p) ; p=1, \ldots, n_{c}\right) \quad$ or $a$ posteriori estimated values $\left(\varepsilon^{\mathrm{o}}(k-p) ; p=1, \ldots, n_{c}\right)$ can be solved this problem.

By equation (29), the a priori prediction error is given by:

$$
\xi(k)=y(k)-\hat{\theta}_{e}^{T}(k-1) \breve{X}(k)
$$

where

$$
\begin{aligned}
\breve{X}(k)= & \Gamma\left(\alpha_{k}\right)^{T} \breve{\varphi}(k) \\
\breve{\varphi}^{T}(k)= & {\left[-y(k-1) \ldots-y\left(k-n_{a}\right) u(k-d-1) \ldots\right.} \\
& \left.u\left(k-d-n_{b}\right) \varepsilon(k-1) \ldots \varepsilon(k-n c)\right]
\end{aligned}
$$

and

$$
\varepsilon(k)=\xi(k)-\zeta^{T}\left(\alpha_{k}\right) \breve{\varphi}(k)
$$

Such that $\hat{\theta}_{e}(k)$ is determined by the modified recursive extended least squares algorithm RELS with relative dead zone, which is defined by:

$$
\begin{aligned}
& \hat{\theta}_{e}(k)=\hat{\theta}_{e}(k-1)+\delta(k) L(k) \xi(k) \\
& L(k)=\frac{P(k-1) \breve{X}(k)}{\mu(k)+\breve{X}^{T}(k) P(k-1) \breve{X}(k)} \\
& P(k)=\left[I-\delta(k) L(k) \breve{X}^{T}(k)\right] P(k-1) \\
& \xi(k)=y(k)-\hat{\theta}_{e}^{T}(k-1) \breve{X}(k) \\
& \varepsilon(k)=\xi(k)-\zeta^{T}\left(\alpha_{k}\right) \breve{\varphi}(k) \\
& \hat{\theta}(k)=\Gamma\left(\alpha_{k}\right) \hat{\theta}_{e}(k)
\end{aligned}
$$

when: 
$\delta(k)= \begin{cases}0 & \text { if }|\xi(k)| \leq \beta d(k) \\ \gamma & \text { otherwise } \in[\sigma, 1 / \beta-\sigma]\end{cases}$

and

$d(k)=\rho\|\breve{\varphi}(k)\|+m_{0}$

\section{SELF-TUNING REGULATOR}

In this section, we study the problem of robust self-tuning regulator for stochastic linear systems with time-varying parameters. This problem can be solved using the minimum variance approach or the generalized minimum variance approach with explicit schemes.

The system described by (1) can be defined by:

$$
\begin{aligned}
y(k+d+1)= & q \frac{B\left(q^{-1}, k\right)}{A\left(q^{-1}, k\right)} u(k)+\frac{G\left(q^{-1}, k\right)}{A\left(q^{-1}, k\right)} e(k) \\
& +F\left(q^{-1}, k\right) e(k+d+1)
\end{aligned}
$$

where $F\left(q^{-1}, k\right)$ and $G\left(q^{-1}, k\right)$ are solution of the following polynomial equation:

$C\left(q^{-1}\right)=A\left(q^{-1}, k\right) F\left(q^{-1}, k\right)+q^{-d-1} G\left(q^{-1}, k\right)$

The polynomials $F\left(q^{-1}, k\right)$ and $G\left(q^{-1}, k\right)$ are given by, respectively:

$F\left(q^{-1}, k\right)=1+f_{1}(k) q^{-1}+\ldots+f_{d}(k) q^{-d}$

and

$G\left(q^{-1}, k\right)=g_{0}(k)+g_{1}(k) q^{-1}+\ldots+g_{n-1}(k) q^{-n+1}$

\subsection{Minimum variance self-tuning regulation}

Formulation of the regulation problem of the considered systems, which are described by ARMAX mathematical model (1), can be formalized by the minimization the following criterion:

$$
J(k+d+1)=\mathrm{E}\left[y^{2}(k+d+1)\right]
$$

where $\mathrm{E}$ denotes the expectation and $y(k+d+1)$ is given by (41), $e(k)$ introduces in (41) can be defined by:

$$
e(k)=\frac{A\left(q^{-1}, k\right)}{C\left(q^{-1}\right)} y(k)-\frac{q^{-d} B\left(q^{-1}, k\right)}{C\left(q^{-1}\right)} u(k)
$$

Now, we can replace $e(k)$ introduces in (41) by (46), we obtain:

$$
\begin{aligned}
y(k+d+1)= & \frac{H\left(q^{-1}, k\right)}{C\left(q^{-1}\right)} u(k)+\frac{G\left(q^{-1}, k\right)}{C\left(q^{-1}\right)} y(k) \\
& +F\left(q^{-1}, k\right) e(k+d+1)
\end{aligned}
$$

where

$$
H\left(q^{-1}, k\right)=q B\left(q^{-1}, k\right) F\left(q^{-1}, k\right)
$$

which can be described as:

$$
H\left(q^{-1}, k\right)=h_{1}(k)+h_{2}(k) q^{-1}+\ldots+h_{n+d}(k) q^{-n-d-1}
$$

$$
\begin{aligned}
J(k+d+1)= & \mathrm{E}\left[\left(\left(H\left(q^{-1}, k\right) / C\left(q^{-1}\right)\right) u(k)\right.\right. \\
& +\left(G\left(q^{-1}, k\right) / C\left(q^{-1}, k\right)\right) y(k) \\
& \left.\left.+F\left(q^{-1}, k\right) e(k+d+1)\right)^{2}\right]
\end{aligned}
$$

We can obtain:

$$
\begin{aligned}
J(k+d+1)= & \left(\left(\frac{H\left(q^{-1}, k\right)}{C\left(q^{-1}, k\right)} u(k)+\frac{G\left(q^{-1}, k\right)}{C\left(q^{-1}, k\right)} y(k)\right)^{2}\right. \\
& +\left(1+f_{1}^{2}(k)+\ldots+f_{d}^{2}(k)\right) \sigma^{2}
\end{aligned}
$$

Then, the optimal control law $u(k)$ which minimizes the criterion (45) is defined by:

$$
u(k)=-\frac{G\left(q^{-1}, k\right)}{H\left(q^{-1}, k\right)} y(k)
$$

where $H\left(q^{-1}, k\right)$ is defined by (48).

The explicit schemes of minimum variance self-tuning regulator can be unrolling considering the three following steps:

Step 1: estimation of parameters intervening in the ARMAX mathematical model (1) (or (29)) using the modified estimation algorithm RELS with relative dead zone given by the set of equation (38). One obtained the polynomials $\hat{A}\left(q^{-1}, k\right), \hat{B}\left(q^{-1}, k\right)$ and $\hat{C}\left(q^{-1}, k\right)$, when:

$$
\begin{aligned}
& \hat{A}\left(q^{-1}, k\right)=\hat{A}\left(q^{-1}, \alpha_{k}\right) \\
& \hat{B}\left(q^{-1}, k\right)=\hat{B}\left(q^{-1}, \alpha_{k}\right)
\end{aligned}
$$

Step 2: determination of the polynomials $F\left(q^{-1}, k\right)$ and $G\left(q^{-1}, k\right)$ by resolving the following polynomial equation:

$$
\hat{C}\left(q^{-1}, k\right)=\hat{A}\left(q^{-1}, \alpha_{k}\right) F\left(q^{-1}, k\right)+q^{-d-1} G\left(q^{-1}, k\right)
$$

Also, the polynomial $H\left(q^{-1}, k\right)$ is calculated using:

$$
H\left(q^{-1}, k\right)=q \hat{B}\left(q^{-1}, \alpha_{k}\right) F\left(q^{-1}, k\right)
$$

Step 3: calculate the control law $u(k)$ by the following equation:

$$
u(k)=-\frac{\sum_{r=2}^{n+d} h_{r}(k)}{h_{1}(k)} u(k-r+1)-\frac{\sum_{s=0}^{n-1} g_{s}(k)}{h_{1}(k)} y(k-s)
$$

Noting that if we have $h_{1}(k)=0$, then we take $h_{1}(k)=0.01$.

\subsection{Generalized minimum variance self- tuning regulation}

Now, we develop the control law which permit to reduce the effect of noise upon the out-put of system, and to limit the control signal amplitude to a desired value. The generalized minimum variance self-tuning regulator can be developed by minimizing the following criterion:

$$
J(k+d+1)=\mathrm{E}\left[y^{2}(k+d+1)+\alpha u^{2}(k)\right]
$$

where $\mathrm{E}$ denotes the expectation and $\alpha$ is a weighting factor given by the user. Then, the optimal control law $u(k)$ which minimizes the criterion (45) is defined by:

The criterion to be minimized becomes: 
$u(k)=-\frac{G\left(q^{-1}, k\right)}{Z\left(q^{-1}, k\right)} y(k)$

where the polynomial $Z\left(q^{-1}, k\right)$ is given by:

$$
Z\left(q^{-1}, k\right)=q B\left(q^{-1}, k\right) F\left(q^{-1}, k\right)+\left(\frac{\alpha}{b_{1}(k)}\right) C\left(q^{-1}\right)
$$

Which can be defined as

$Z\left(q^{-1}, k\right)=z_{1}(k)+z_{2}(k) q^{-1}+\ldots+z_{n+d}(k) q^{-n-d+1}$

The explicit schemes of generalized minimum variance selftuning regulator can be unrolling considering the three following steps:

Step 1: estimation of parameters intervening in the ARMAX mathematical model (1) (or (29)) using the modified estimation algorithm RELS with relative dead zone given by the set of equation (38);

Step 2: determination of the polynomials $F\left(q^{-1}, k\right)$ and $G\left(q^{-1}, k\right)$ by resolving the polynomial equation (55);

Also, he polynomial $Z\left(q^{-1}, k\right)$ is calculated using:

$$
Z\left(q^{-1}, k\right)=q \hat{B}\left(q^{-1}, \alpha_{k}\right) F\left(q^{-1}, k\right)+\left(\frac{\alpha}{\hat{b}_{1}\left(\alpha_{k}\right)}\right) \hat{C}\left(q^{-1}, k\right)
$$

Step 3: calculate the control law $u(k)$ by the following equation:

$$
u(k)=-\frac{\sum_{r=2}^{n+d} z_{r}(k)}{z_{1}(k)} u(k-r+1)-\frac{\sum_{s=0}^{n-1} g_{s}(k)}{z_{1}(k)} y(k-s)
$$

Noting that if we have $z_{1}(k)=0$, then we take $z_{1}(k)=0.01$.

we uses a numerical example, which permits to compare performances of the algorithm of minimum variance output self-tuning regulation basing on the modified recursive extended least squares estimation algorithm with a relative dead zone and the algorithm of minimum variance self-tuning regulation basing on the recursive extended least squares estimation algorithm with exponential forgetting factor treated in the literature([3], [8], [10], [11], [12], [14], [15] [18],[23]), to demonstrate the robustness of the minimum variance output self tuning regulator basing on the modified RELS estimation algorithm.

The RELS estimation algorithm with exponential forgetting factor is given by:

$$
\begin{aligned}
\hat{\theta}(k) & =\hat{\theta}(k-1)+P(k) \breve{\varphi}(k) \varepsilon(k) \\
P(k) & =\frac{1}{\lambda(k)}\left[P(k-1)-\frac{P(k-1) \breve{\varphi}(k) \breve{\varphi}^{T}(k) P(k-1)}{\lambda(k)+\breve{\varphi}^{T}(k) P(k-1) \breve{\varphi}(k)}\right] \\
\varepsilon(k) & =y(k)-\hat{\theta}^{T}(k-1) \breve{\varphi}(k)
\end{aligned}
$$

when $\breve{\varphi}(k)$ is defined by (36) and $\lambda(k), 0<\lambda(k)<1$, is an exponential forgetting factor, which can be defined by the following equation:

$\lambda(k)=\lambda_{\mathrm{o}} \lambda(k-1)+\lambda^{\mathrm{o}}\left(1-\lambda_{\mathrm{o}}\right)$

where: $0<\lambda_{o}<1,0<\lambda^{o}<1$.

\section{NUMERICAL EXAMPLE}

In this section, we study the problem of self-tuning regulator for a linear stochastic system, which can be described by discreet time input-output mathematical model ARMAX with unknown time-varying parameters. In this case, we will use an explicit scheme of self-tuning regulation with minimum variance. The noise acting on the system is a sequence of independent random variables with zero mean and constant variance.

The consider system can be described by the following mathematical model ARMAX:

$$
y(k)=-a_{1}(k) y(k-1)+b_{1}(k) u(k-2)+e(k)+c_{1} e(k-1)
$$

In the mathematical model ARMAX (65), the sequences $\{e(k)\}$ is a white noise with zero means and constant variances. We suppose that the parameters of the system are unknown and the order and delay are known exactly.

The developed explicit self-tuning regulator with the modified RELS estimation algorithm with a relative dead zone is used to regulate the considered system. The parameters of this model are supposed to be unknown and time-varying, which are chosen as:

$$
a_{1}(k)=0.5+0.2 \frac{1+k}{1+2 k} \cos \left(\frac{3.14}{40} k\right), b_{1}(k)=0.4, c_{1}=0.1
$$

The parameter vector is defined by:

$\theta^{T}(k)=\left[a_{1}(k) b_{1}(k) c_{1}\right]$

The number of data was 300

The control law $u(k)$ is computed on the basis upon the minimization of the following criteria:

$$
J(k+2)=\mathrm{E}\left[y^{2}(k+2)\right]
$$

The steps of computing the control law $u(k)$ of self-tuning regulator in the minimum variance self-tuning regulation algorithm are based on the modified recursive extended least squares estimation algorithm with a relative dead zone are listed in the following:

Steps 1: Calculate the estimated parameters intervening in the ARMAX mathematical model (65) using the modified RELS estimation algorithm with a relative dead zone given by the set of equation (38). In this step we take:

1. $e(k)$ is white noise with a zero means and variance $\sigma^{2}=0.138^{2}$

2. the orders of limited development are: $N_{1}=1, M_{1}=0$;

3. the initial values of the estimated parameters $\hat{\theta}_{e}(0)$ and the gain matrix $P(0)$ are chosen as: $\hat{\theta}_{e}(i)=0, i \leq 0$, $P(0)=1000 I$, where $I$ denote the identity matrix, $y(i)=0, u(i)=0, i \leq 0$;

4. $\beta=2, \mu(k)=0.98$ and $\gamma=0.44$.

The estimated parameters vector $\hat{\theta}_{e}(k)$ is given by:

$\hat{\theta}_{e}^{T}(k)=\left[\begin{array}{lll}\hat{\theta}_{a_{1}}^{T}(k) & \hat{b}_{1}(k) & \hat{c}_{1}(k)\end{array}\right]$

The estimated parameter $\hat{a}_{1}(k)$ is given by:

$\hat{a}_{1}(k)=J_{N 1}\left(\alpha_{k}\right) \hat{\theta}_{a_{1}}(k)$

where $\alpha_{k}$ has been defined in [5] 
Step 2: determination of the polynomials parameter $F\left(q^{-1}, k\right)$ and $G\left(q^{-1}, k\right)$ by resolving the polynomial equation (55), where: $F\left(q^{-1}, k\right)=1+f_{1}(k) q^{-1}, \quad G\left(q^{-1}, k\right)=g_{0}(k)$. The parameters $f_{1}(k)$ and $g_{0}(k)$ are given by: $f_{1}(k)=\hat{c}_{1}(k)-\hat{a}_{1}(k), \quad g_{0}(k)=-\hat{c}_{1}(k) f_{1}(k) . \quad$ The polynomial $H\left(q^{-1}, k\right)$ is calculated using equation (56), where: $\quad H\left(q^{-1}, k\right)=h_{1}(k)+h_{2}(k) q^{-1}$. The parameters $h_{1}(k)$ and $h_{2}(k)$ are given by: $h_{1}(k)=\hat{b}_{1}(k)$, $h_{2}(k)=\hat{b}_{1}(k) f_{1}(k)$;

Step 3: calculate the control law $u(k)$ by the equation (57), where:

$$
u(k)=\left(-\frac{1}{h_{1}(k)}\right)\left(h_{2}(k) u(k-1)+g_{0}(k) y(k)\right)
$$

If we have $\hat{b}_{1}(k)=0$, then we take $h_{1}(k)=0.01$.

The control law of the minimum variance self-tuning regulator, which basing on the modified RELS estimation algorithm with a relative dead zone, and the output of system are shown in Fig. 1. The control law of the minimum variance self-tuning regulator, which basing on the RELS estimation algorithm with an exponential forgetting factor given by (64), and the output of system are shown in Fig. 2. Fig. 3 represent the estimated parameter $\hat{a}_{1}(k)$ and $\hat{b}_{1}(k)$, when the black line represent the real value, the blue line represent the estimated parameter by applying the modified RELS estimation algorithm with a relative dead zone and the red line represent the estimated parameter by applying the RELS estimation algorithm with an exponential forgetting factor. In Fig. 4 we showed the estimated parameter $\hat{c}_{1}(k)$ and the variance of prediction error.

It is observed and shown in Fig. 3 that the proposed modified RELS estimation algorithm with a relative dead zone converge and ensures that the estimated parameter follow the time-varying parameter than the estimated parameter by the RELS estimation algorithm with an exponential forgetting factor.

The control law of the minimum variance self-tuning regulator, which basing on modified RELS estimation algorithm with a relative dead zone, permits to eliminate the noise effect on the system and to remedy the transient regime effect.

We conclude that the robustness of the minimum variance self-tuning regulator is ensured basing on the modified RELS estimation algorithm with a relative dead zone. This example shows the performances of the developed schemes of the minimum variance self-tuning regulation.
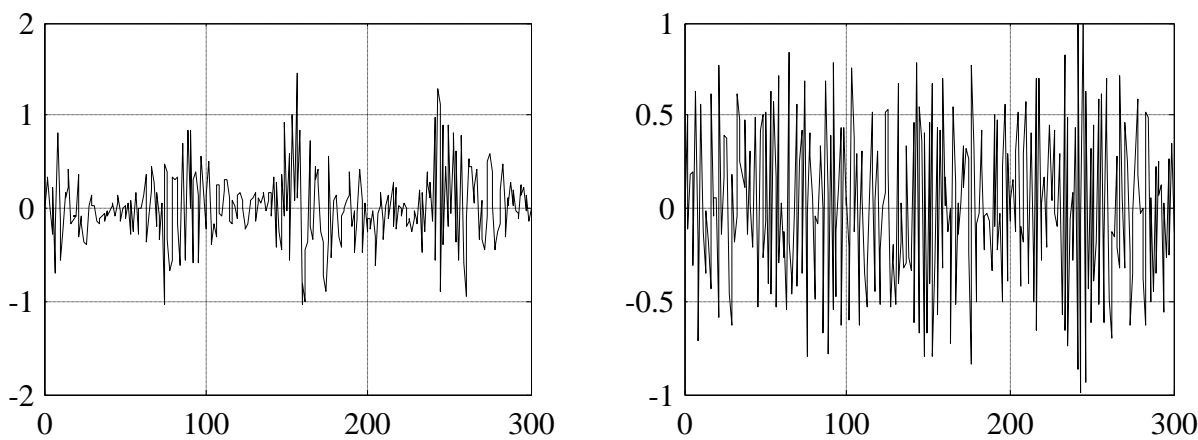

Fig. 1: Control law $u(k)$ and output of system $y(k)$ basing on the modified RELS estimation algorithm with a relative dead zone.
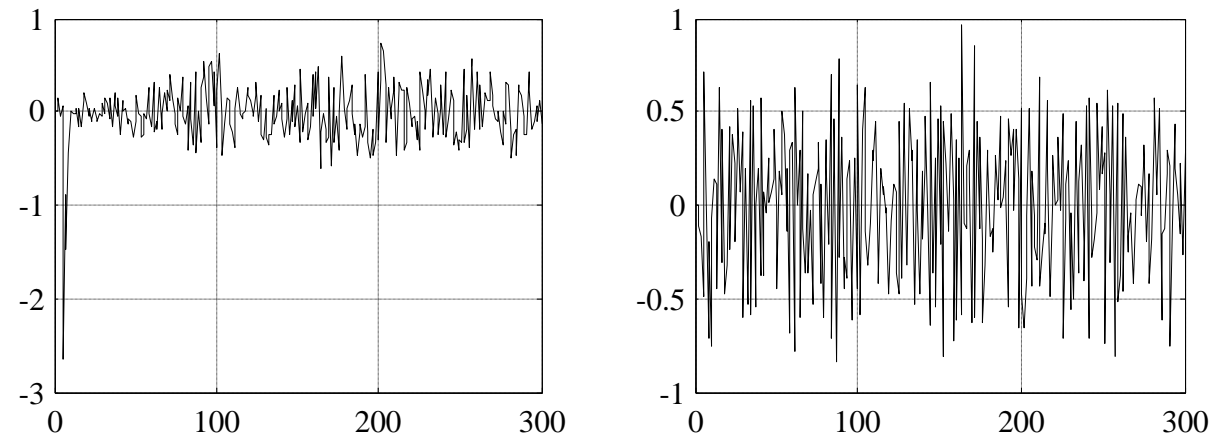

Fig. 2: Control law $u(k)$ and output of system $y(k)$ basing on the RELS estimation algorithm with exponential forgetting factor. 

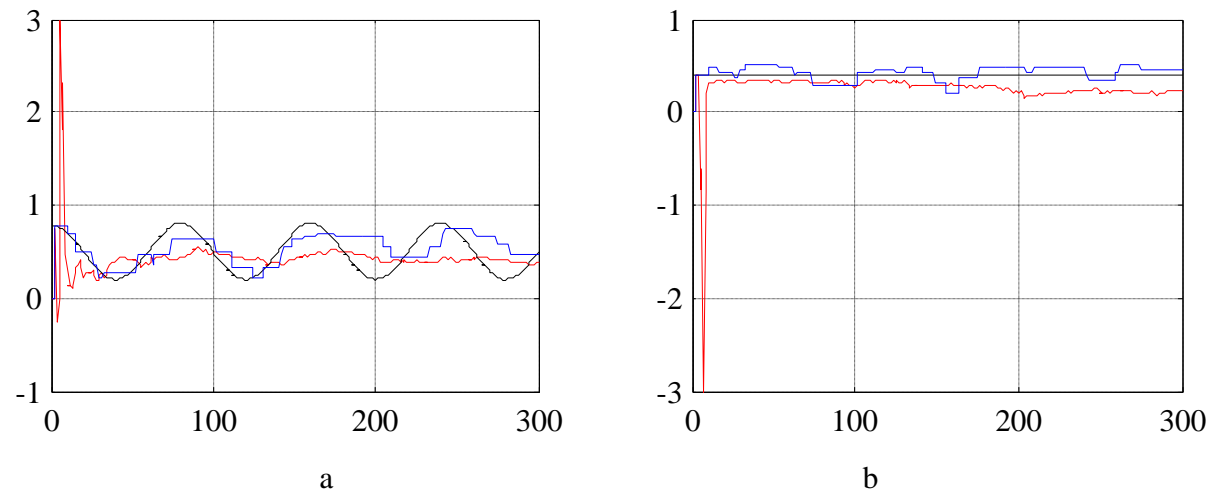

Fig. 3: Estimated parameters of system: a: $\hat{a}_{1}(k), \mathbf{b}: \hat{b}_{1}(k)$

- real value

_ basing on the modified RELS estimation algorithm with a relative dead zone

basing on the RELS estimation algorithm with an exponential forgetting factor
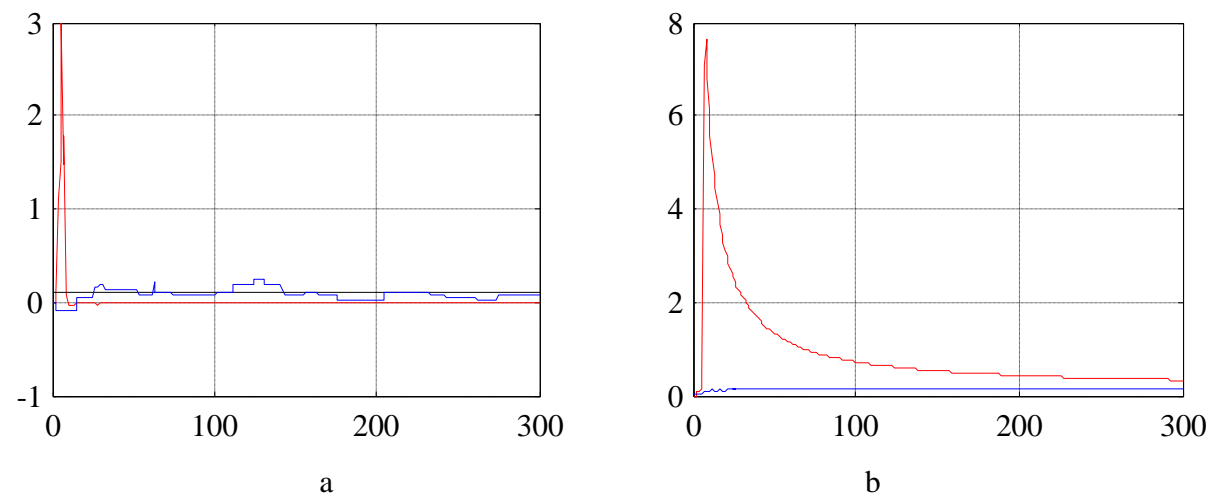

Fig. 4: a: Estimated parameter $\hat{c}_{1}(k)$, b: the variance of prediction error

\section{real value}

_ basing on the modified RELS estimation algorithm with a relative dead zone

\section{basing on the RELS estimation algorithm with an exponential forgetting factor}

\section{CONCLUSION}

The problem of the parametric estimation of the linear dynamic systems, which can be described by input-output ARMAX mathematical models with unknown and timevarying parameters, has been landed in this paper. A schema of polynomial approximation was applied to approximate time-varying parameters. This approach permits to determine the relative dead zone. The modified recursive least squares estimation algorithm $R L S$ with dead zone cannot estimate parameters of systems. Then, a modified recursive extended least squares estimation algorithm RELS with a relative dead zone has been proposed and developed to solve this problem.

The problem of the explicit schemes of robust self-tuning regulator of linear dynamic systems with time-varying parameters is developed. This problem was solved by using the minimum variance output or the generalized minimum variance approach basing on the modified recursive extended least squares estimation algorithm with a relative dead zone.
A Numerical simulation example has been studied. A comparative study, between the explicit schemes algorithm of self-tuning regulator basing on the RELS estimation algorithm with an exponential forgetting factor and the explicit schemes algorithm of self-tuning regulator basing on the modified RELS estimation algorithm with a relative dead zone, shows the effectiveness of the developed control law. This show the performances and the robustness of the explicit schemes of minimum variance self-tuning regulator basing on the proposed modified RELS estimation algorithm with a relative dead zone.

\section{REFERENCES}

[1] Åström K.J. and Wittenmark B. (1973): On self-tuning regulators, Automatica, Vol. 9, pp.185-199.

[2] Clarke D.W. and Gawthrop P.J. (1979): Self-tuning control. Proceedings of IEE, Vol. 126, pp. 633-640. 
[3] Fortescue T.R., Kershenbaum L.S. and Ydstie B.E. (1981): Implementation of Self Tuning Regulators with Variable Forgetting Factors, Automatica, 27, pp. 831-835.

[4] Goodwin G.C., Elliot H. and Teoh E.K. (1983): Deterministic convergence of a self-tuning regulator with covariance resetting. IEE Proc. D Control Theory and Appl, 130, pp. 6-8.

[5] Gu X.Y. and Shao C. (1993): Robust adaptive control of time-varying linear plants using polynomial approximation. IEE Proceedings-D, vol.140, pp. 111118.

[6] Isermann R. (1984): Process fault detection based on modeling and estimation methods a survey. Automatica, 20(4):pp. 387-404.

[7] Isermann R. (2005): Model-based fault-detection and diagnosis - status and applications. Annual Reviews in Control, 29(1):pp. 71-85.

[8] Jiang J. and Youmin Z. (2004): A revisit to block and recursive least squares for parameter estimation. Computers and Electrical Engineering, 30, 403-416.

[9] Johansson R. (1993): System Modeling and Identification. Prentice Hall, Englewood Cliffs.

[10] Kamoun M. and Titli A. (1988): Parametric identification of large scale discrete time systems. Information and Decision Technologies, Vol. 14, pp. 289-306.

[11] Kamoun S. (2003) : Contribution à l'identification et à la commande adaptative de systèmes complexes. Thesis of doctoral in automatic control. National School of Engineering of Sfax, University of Sfax, 246 pages.

[12] Li Z. (1988): Discrete-time adaptive control for timevarying systems subject to unknown fast time-varying deterministic disturbances, IEE Proc. D, Control theory \& Appl., 135, pp. 445-450.
[13] Li Z. and Evans R.J. (2002): Generalized minimum variance control of linear time-varying systems. IEE Proc. Control Theory Appl., Vol. 149, pp. 111-116.

[14] Ljung L. and Söderström T. (1983): Theory and Practice of Recursive Identification. MIT Press, Cambridge, Massachusetts.

[15] Maciej N. Z. and Tomasz K. (2001): Fast recursive basis function algorithms for identification of time-varying processes. Proc. IEEE Conf. Decision Contr, 40, pp. 4307-4302.

[16] Ohkawa F. (1986): MRAC for discrete time-varying systems with periodically varying parameters and time delay. Int. J. Control, 44, pp 171-179.

[17] Thil S. (2007): Contributions à l'identification de modèles avec des erreurs en les variables. Thesis of Doctorat, University of Henri Poincaré, Nancy 1.

[18] Toplis B. and Pasupathy S. (1988): Tracking improvements in fast RLS algorithms using a variable forgetting factor. IEEE Trans Acoust Speech Signal Process;ASSP-36(2):206-27.

[19] Tsakalis K. and Ioannou P.A. (1987): Adaptive control of linear time-varying plants. Automatica, 23, pp 459-468.

[20] Van den Hof P. (1996): System identification. Technical Report.

[21] Xianya X. and Evens R. (1984): Discrete-time stochastic adaptive control for time-varying systems. IEEE Trans, $A C-29$, pp. 638-641.

[22] Xiukun W., Luigi D.R. and Jindong T. (2005): Robust adaptive control of quasi-LPV systems. Proc. IEEE Conf. Advanced Intelligent Mechatronics. pp. 1617-1622.

[23] Ydstie B.E. (1985): Adaptive Control and Estimation with Forgetting Factors, 7th IFAC Symposium on Identification and System Parameter Estimation, York, July England. 